\title{
Assessment of Success Rate of Directly Observed Treatment Short-Course (DOTS) in Tuberculosis Patients of South India
}

\author{
Ramakrishna Prudhivi" ${ }^{*}$, Siva Reddy Challa², Basaveswara Rao MV³, Veena G4, Bhaskara Rao N5, Hema Manogna Narne ${ }^{6}$ \\ 'Department of Pharmacy Practice, Faculty of Pharmacy, Krishna University, Machilipatnam- 521001, Andhra Pradesh, INDIA. \\ ${ }^{2}$ Department of Pharmacology, KVSR Siddhartha College of Pharmaceutical Sciences, Siddhartha Nagar, Vijayawada-520010, Andhra Pradesh, INDIA. \\ ${ }^{3}$ Department of Chemistry, Faculty of Pharmacy, Krishna University, Machilipatnam-521001, Andhra Pradesh, INDIA. \\ ${ }^{4}$ Department of Pharmacology, Faculty of Pharmacy, Krishna University, Machilipatnam-521001, Andhra Pradesh, INDIA. \\ ${ }^{5}$ Department of Tuberculosis and Chest Diseases, Guntur Govt. ID Hospital, Guntur-522002, Andhra Pradesh, INDIA. \\ 'Department of Pharmacy Practice, Hindu College of Pharmacy, Guntur-522002, Andhra Pradesh, INDIA.
}

\begin{abstract}
Objective: To assess the success rate of DOTS for tuberculosis patients and the secondary objective was to identify the factors associated with unsuccessful treatment outcome. Methods: A retrospective study was conducted to review the medical records of patients $(n=1113)$ registered at the Directly Observed Treatment Short-Course (DOTS) clinic of Government Infectious Disease (Govt. ID) Hospital, Guntur, India. Multivariate logistic regression model was used to determine the factors associated with the treatment success rate. Results: The overall mean success rate of TB patients was found to be $82.8 \%$. Treatment success rate (TSR) was steadily increased across the years from $73.9 \%$ in 2015 to $84.3 \%$ in 2016 and $88.9 \%$ in 2017 while the death rate was steadily decreased from $11.2 \%$ in 2015 to $6.25 \%$ in 2016 and $4.33 \%$ in 2017 . Risk factors significantly associated with unsuccessful treatment outcome were found to be HIV positive $(P<0.001)$, smear negative $(P<0.001)$, all retreatment cases $(P<0.001)$, smoking $(P=0.008)$, and alcoholism $(P=0.019)$. Smear positive patients had lower death rate $(3.9 \%$ vs. $10.1 \% ; P<0.001)$ and failure rate ( $(2.6 \%$ vs. $8.7 \% ; P<0.001)$ compared to smear negative patients. Patients tested HIV positive had seen significantly unfavorable outcomes
\end{abstract}

in death rate $(\mathrm{OR}=9.17,95 \% \mathrm{Cl}=5.31-15.83 ; P<0.001)$ and treatment failure $(\mathrm{OR}=13.3,95 \% \mathrm{Cl}=7.31-24.17 ; P<0.001)$. Conclusion: Implementing the DOTS strategy proved the satisfactory success rate in the South Indian hospital across three years. The unsuccessful treatment outcome was significantly associated with gender, HIV status, re-treatment, smear negative, smoking and alcoholism.

Key words: Directly observed treatment short-course, Retrospective study, Risk factors, Treatment success rate, Tuberculosis.

\section{Correspondence}

Mr. Ramakrishna Prudhivi, M.Pharm, (Ph. D), Faculty of Pharmacy, Department of Pharmacy Practice, Krishna University, Machilipatnam- 521001, Andhra Pradesh, INDIA

Phone: +91 8618194642

Email: ramakrishna.prudhivi@gmail.com

DOI: 10.5530/jyp.2019.11.14

\section{INTRODUCTION}

Tuberculosis (TB) has existed for millennia and remains a major global health problem. It causes ill-health in millions of people each year. In 2015 , it was one of the top ten causes of death worldwide, ranking above HIV/AIDS as one of the leading causes of death from an infectious disease. Six countries accounted for $60 \%$ of the new cases: India, Indonesia, China, Nigeria, Pakistan and South Africa. ${ }^{1}$

In 2016, there were an estimated 1.3 million TB deaths among HIVnegative people and an additional 374000 deaths among HIV-positive people. India accounted for $33 \%$ of global TB deaths among HIVnegative people. Globally, the TB mortality rate is falling at about $3 \%$ per year. TB incidence is falling at about $2 \%$ per year and $16 \%$ of TB cases die from the disease; by 2020 , these figures need to improve to $4-5 \%$ per year and $10 \%$, respectively to reach the first (2020) milestones of the End TB Strategy. ${ }^{2}$

In India, $29 \%$ of adults but $72 \%$ of HIV-positive adults live in four large states in the south where, even with the RNTCP, mortality is expected to fall by only $15 \%$ between 1990 and $2015 .{ }^{3}$ There are more challenges for controlling TB worldwide by adopting the medication adherence. There was a high level of adherence to anti-TB treatment and also a high TB treatment success rate. ${ }^{4}$
Countries implementing DOT to ensure treatment adherence have shown impressive results with increasing treatment success and low default rates. ${ }^{1,5,6}$ India has had an ongoing National TB Program (NTP) since $1962 . .^{7}$ Large-scale implementation of the RNTCP began in late $1998 .^{8}$ Though the Indian government has made several announcements to eliminate TB by 2025 , the WHO report showed that up to 27.9 lakh patients were estimated to be infected in the country in 2016. Out of the 27.9 lakh estimated patients, only $1,938,158$ TB cases were notified in the public and private sector in India, which means over 8.5 lakh cases were missing the treatment options.

In order to overcome these lacunae, the Government decided to give a new thrust to TB control activities by revitalizing the NTP, with assistance from international agencies, in 1993. The Revised National Tuberculosis Control Programme (RNTCP) thus formulated, adopted the internationally recommended Directly Observed Treatment Short-course (DOTS) strategy, as the most systematic and cost-effective approach to revitalize the TB control programme in India.

Directly observed alternate day treatment (DOTS) for TB under RNTCP in India has shown to be effective in TB patients with or without HIV infection. ${ }^{9} 10$ Furthermore, World Health Organization (WHO) has

This is an open access article distributed under the terms of the Creative Commons Attribution-NonCommercial-ShareAlike 4.0 License, which allows others to remix, tweak, and build upon the work non-commercially, as long as the author is credited and the new creations are licensed under the identical terms. 
recommended the use of standard short course therapy for active TB in HIV positive patients. ${ }^{11}$

Monitoring the treatment outcome using standardized approach is required to evaluate the effectiveness of the intervention. However, treatment outcome has not been assessed yet in South Indian population visiting Govt. ID hospital, Guntur, India Guntur, India. Therefore, this study aimed to assess treatment outcome of all type of TB patients over 3 -year period retrospectively.

\section{METHODOLOGY}

\section{Ethics Approval and Consent to Participate}

All participants gave written informed consent to participate. Permission to conduct the study as obtained from Government infectious Disease Hospital, Guntur, India. The study was approved by the Institutional Ethics Committee of Government infectious Disease Hospital, Guntur, India.

\section{Study design, study settings and data collection}

This was a retrospective cohort study of pulmonary tuberculosis patients registered in DOTS centre of Govt. Infectious Disease Hospital, Guntur, India from January, 2014 to December 2016 and for their treatment outcome from 2015 to 2017. All participants gave written informed consent and the study was approved by the Ethics Committee of Government Infectious Disease Hospital, Guntur, India.

The data collected from TB patients medical records included sociodemography, habits of patient, type of TB, history of TB, HIV status, treatment initiation and it's follow up, outcomes of TB. All the patient data retrieved from DOTS centre. All patients of either sex suffering from pulmonary TB were included in the study. We enrolled new and retreatment cases that were recorded during the study period. New cases involved all untreated smear positive pulmonary $\mathrm{TB}$ and smear negative pulmonary TB with extensive parenchymal involvement. Retreatment cases included all relapse, failure, transferred in and return after default. All other forms of TB (extra pulmonary), like military TB, TB pleurisy, hilar and/or mediastinal lymphadenopathy, spinal, intestinal and genitourinary TB were excluded. Some patient's records were excluded due to incomplete information about treatment outcome and transferred out to other health care clinics after the declination of the prescribed treatment (Figure 1).

\section{Data analysis}

Primary outcome measure was the success rate of DOTS for tuberculosis patients and secondary outcome measures were the factors associated with unsuccessful treatment outcome. Patients were followed up to the end of treatment. According to WHO and International Union Against Tuberculosis and Lung Disease (IUATLD) guidelines, treatment outcomes were categorized as cured (finished treatment with negative smear or culture test at the end of the treatment), completed (finished treatment but does not have negative sputum smear or culture at the end of the treatment), treatment failure(sputum or culture test is positive after 5 months), default (patients who interrupted their treatment for two consecutive months or more after treatment commencement), died (patients who die of any reason during the course of treatment) and transferred out (patients who transferred to another healthcare units and whose treatment outcome is unknown). Treatment success rate is calculated as sum of cured and treatment completed.

\section{Statistical analysis}

Categorical variables were reported as frequency and percentage frequency. Data was mentioned in mean \pm SD values. Chi square test was performed to determine the strength of association between that
Table 1: Patient recruitment for period of 2014-2016.

\begin{tabular}{ccccc}
\hline \multirow{2}{*}{ Method } & \multicolumn{3}{c}{ Yearly Distribution } & Distribution in 3yrs \\
\cline { 2 - 4 } & 2014 & $\mathbf{2 0 1 5}$ & $\mathbf{2 0 1 6}$ & (\%) \\
\hline New & $194(56)$ & $239(67.8)$ & $328(79)$ & $761(68.3)$ \\
Relapse & $22(6.3)$ & $16(4.5)$ & $32(7.7)$ & $70(6.28)$ \\
Failure & $16(4.6)$ & $28(7.9)$ & $15(3.6)$ & $59(5.3)$ \\
Transferred in & $26(7.5)$ & $18(5.1)$ & $8(1.9)$ & $52(4.6)$ \\
Return after & $88(25.4)$ & $51(14.4)$ & $32(7.7)$ & $171(15.3)$ \\
default & & & & \\
TOTAL & $\mathbf{3 4 6 ( 3 1 . 1 )}$ & $\mathbf{3 5 2 ( 3 1 . 6 )}$ & $\mathbf{4 1 5 ( 3 7 . 2 )}$ & $\mathbf{1 1 1 3 ( 1 0 0 )}$ \\
\hline
\end{tabular}

outcome and variables and crude odds ratio (COR) was determined. In addition, multivariate logistic regression analysis was applied to determine the factors affecting the treatment success rate following adjusting for all confounding variables and adjusted Odds ratio (AOR) were calculated. P-value less than 0.001 were considered as statistically significant. Data was analyzed using Epi info version.7 statistical tool and Graph Pad Prism software version 5.0.

\section{RESULTS}

\section{Patient characteristics}

A total of 1113 pulmonary tuberculosis patients had attended DOTS centre at Govt. ID hospital, Guntur, India between January 2015 and December 2017. Patients were recruited through different methods: New, Relapse, Failure, Transferred in and Return after default. The majority $761(68.3 \%)$ of the patients were new TB cases whereas $70(6.28 \%)$ were relapse cases; 59(5.3\%) were treatment failure cases; 52 (4.6\%) were transferred in cases; and $171(15.3 \%)$ were return after default. Total retreatment cases were $352(31.6 \%)$ cases (Table 1 ).

Out of 1113 TB patients, 734(66\%) were males while the remaining were females. The age of the patients ranged from 16 to 80 years with a mean (SD) of $47.13(14.5)$ years. The majority $284(25.5 \%)$ of the patients belonged to age group 50-59 years. Rural patients made up 948(85.1\%) of the cases, while $165(14.8 \%)$ were from urban. Sixty eight percent (756) of the patients had sputum test positive and remaining had negative results. Overall, 23\% (256) of the patients had a history of Human Immunodeficiency Virus (HIV) positive. There were 53.5\% (596) cases had a history of smoking and 35.3\% (393) had a habit of alcohol consumption. The socio demographic and clinical characteristics of tuberculosis patients included in the study are shown in Table 2.

\section{Treatment outcomes}

Out of $1113,82.8 \%$ (922) of the patients were successfully treated (cured and treatment completed), 5.7\% (64) were treatment failure, 5.9\% (66) were died, $4.5 \%$ (51) were default, $0.9 \%$ (10) were transferred out. Treatment success rate was steadily increased across the years from $73.9 \%$ in 2015 to $84.3 \%$ in 2016 and $88.9 \%$ in 2017 while the death rate was steadily decreased from $11.2 \%$ in 2015 to $6.25 \%$ in 2016 and $4.33 \%$ in 2017 (Table 3).

Higher default rates were observed in elderly age group, male patients, patients living in rural areas, smear negative, HIV negative, default, patients having smoking habit and alcohol consumption. Factors associated with higher treatment failure rates included being male, elderly age group, living in rural areas, smear negative, HIV positive, treatment failure, patients with smoking habit and alcohol consumption. The death rate was found be high in elderly age group, being male, living in rural areas, smear negative, HIV positive, transferred in, patients having smoking habit and alcohol consumption. 
Table 2: Socio-demographic and clinical characteristics of tuberculosis patients.

\begin{tabular}{|c|c|}
\hline Variables & $\mathrm{N}(\%)$ \\
\hline \multicolumn{2}{|l|}{ Gender } \\
\hline Male & $734(66)$ \\
\hline Female & $379(34)$ \\
\hline \multicolumn{2}{|l|}{ Age (years) } \\
\hline$<30$ & 101(8.9) \\
\hline $30-39$ & $234(21)$ \\
\hline $40-49$ & $239(21.4)$ \\
\hline $50-59$ & $284(25.5)$ \\
\hline$\geq 60$ & $255(22.9)$ \\
\hline \multicolumn{2}{|l|}{ Education } \\
\hline Illiterate & $162(14.5)$ \\
\hline School & $840(75.5)$ \\
\hline Pre-university & $52(4.6)$ \\
\hline University & $59(5.3)$ \\
\hline \multicolumn{2}{|l|}{ Occupation } \\
\hline Business & $48(4.3)$ \\
\hline Private & $525(47.2)$ \\
\hline Agriculture & $204(18.3)$ \\
\hline Old aged & $245(22)$ \\
\hline Govt & $67(6.0)$ \\
\hline Student & $24(2.1)$ \\
\hline \multicolumn{2}{|l|}{ Residence } \\
\hline Urban & $165(14.8)$ \\
\hline Rural & $948(85.1)$ \\
\hline \multicolumn{2}{|l|}{ Marital status } \\
\hline Married & $935(84)$ \\
\hline unmarried & $116(10.4)$ \\
\hline widowed & $47(4.2)$ \\
\hline divorced & $15(1.3)$ \\
\hline \multicolumn{2}{|l|}{ Smoking } \\
\hline Yes & $596(53.5)$ \\
\hline No & $517(46.4)$ \\
\hline \multicolumn{2}{|l|}{ Alcohol } \\
\hline Yes & $393(35.3)$ \\
\hline No & $720(64.7)$ \\
\hline \multicolumn{2}{|l|}{ HIV status } \\
\hline Positive & $256(23)$ \\
\hline Negative & $857(77)$ \\
\hline \multicolumn{2}{|l|}{ Smear test } \\
\hline positive & $756(67.9)$ \\
\hline negative & $357(32)$ \\
\hline \multicolumn{2}{|c|}{ History of TB treatment } \\
\hline New cases & $761(68.3)$ \\
\hline retreatment & $352(31.6)$ \\
\hline \multicolumn{2}{|c|}{ Treatment outcome } \\
\hline Cured & $659(59.2)$ \\
\hline Completed & $263(23.6)$ \\
\hline Died & $66(5.9)$ \\
\hline Failure & $64(5.7)$ \\
\hline Default & $51(4.5)$ \\
\hline Transfer out & $10(0.9)$ \\
\hline
\end{tabular}

Table 3: Treatment outcome for 2015, 2016 and 2017.

\begin{tabular}{ccccc}
\hline \multirow{2}{*}{$\begin{array}{c}\text { Treatment } \\
\text { outcome }\end{array}$} & \multicolumn{2}{c}{ Yearly distribution of patients } & \begin{tabular}{c} 
Percentage \\
\cline { 2 - 4 } rate in 3yrs (\%)
\end{tabular} \\
\cline { 2 - 4 } Cure & $\mathbf{2 0 1 5}$ & $\mathbf{2 0 1 6}$ & $\mathbf{2 0 1 7}$ & $659(59.2)$ \\
Completed & $82(23.7)$ & $74(21.0)$ & $107(25.7)$ & $263(23.6)$ \\
Success Rate & $\mathbf{2 5 6 ( 7 3 . 9 )}$ & $\mathbf{2 9 7 ( 8 4 . 3 )}$ & $\mathbf{3 6 9 ( 8 8 . 9 )}$ & $\mathbf{9 2 2 ( 8 2 . 8 )}$ \\
Treatment failure & $39(11.3)$ & $5(1.4)$ & $20(4.8)$ & $64(5.7)$ \\
Died & $26(7.5)$ & $22(6.25)$ & $18(4.3)$ & $66(5.9)$ \\
Default & $21(6.1)$ & $22(6.25)$ & $8(1.9)$ & $51(4.5)$ \\
Transferred out & $4(1.1)$ & $6(1.7)$ & 0 & $10(0.9)$ \\
Total & $346(31.1)$ & $352(31.6)$ & $415(37.2)$ & $1113(100)$ \\
\hline
\end{tabular}

Table 4: Treatment outcome by socio-demographic and clinical characteristics.

\begin{tabular}{|c|c|c|c|c|c|c|c|}
\hline VARIABLES & $\begin{array}{c}\text { Total TB } \\
\text { cases }\end{array}$ & $\begin{array}{l}\text { Cured } \\
\text { n (\%) }\end{array}$ & $\begin{array}{c}\text { Completed } \\
\text { n (\%) }\end{array}$ & $\begin{array}{c}\text { Failure } \\
\text { n (\%) }\end{array}$ & $\begin{array}{c}\text { Default } \\
\text { n (\%) }\end{array}$ & $\begin{array}{l}\text { Died } \\
\text { n (\%) }\end{array}$ & $\begin{array}{c}\text { Tranfer } \\
\text { out n } \\
(\%)\end{array}$ \\
\hline & 1113 & $659(59.2)$ & $263(23.6)$ & $64(5.7)$ & $51(4.5)$ & $66(5.9)$ & $10(0.9)$ \\
\hline \multicolumn{8}{|l|}{$\begin{array}{c}\text { AGE } \\
\text { (YEARS) }\end{array}$} \\
\hline$<30$ & $101(8.9)$ & $57(56.4)$ & $33(32.7)$ & $3(2.9)$ & $2(1.9)$ & $1(0.9)$ & $5(4.9)$ \\
\hline $30-39$ & $234(21)$ & $139(59.4)$ & $59(25.2)$ & $12(5.1)$ & $5(2.1)$ & $19(8.1)$ & 0 \\
\hline $40-49$ & $239(21.4)$ & $124(51.9)$ & $78(32.6)$ & $13(5.4)$ & $12(5.02)$ & $11(4.6)$ & $1(0.4)$ \\
\hline $50-59$ & $284(25.5)$ & $202(71.1)$ & $37(13)$ & $17(5.9)$ & $14(4.9)$ & $14(4.9)$ & 0 \\
\hline$\geq 60$ & $255(22.9)$ & $137(53.7)$ & $56(21.9)$ & $19(7.4)$ & $18(7)$ & $21(8.2)$ & $4(1.5)$ \\
\hline \multicolumn{8}{|l|}{ GENDER } \\
\hline Male & $734(66)$ & $417(56.8)$ & $171(23.3)$ & $47(6.4)$ & $41(5.6)$ & $51(6.9)$ & $7(0.9)$ \\
\hline Female & $379(34)$ & $242(63.8)$ & $92(24.2)$ & $17(4.5)$ & $10(2.6)$ & $15(3.9)$ & $3(0.8)$ \\
\hline \multicolumn{8}{|l|}{ RESIDENCE } \\
\hline Urban & $165(14.8)$ & $98(59.3)$ & $45(27.2)$ & $8(4.8)$ & $6(3.6)$ & $6(3.6)$ & $2(1.2)$ \\
\hline Rural & $948(85.1)$ & $561(59.1)$ & $218(22.9)$ & $56(5.9)$ & $45(4.7)$ & $60(6.3)$ & $8(0.8)$ \\
\hline \multicolumn{8}{|l|}{$\begin{array}{c}\text { TYPE OF } \\
\text { TB }\end{array}$} \\
\hline $\begin{array}{l}\text { Smear } \\
\text { positive }\end{array}$ & $756(67.9)$ & $476(62.9)$ & 197(26.1) & $27(3.6)$ & $20(2.6)$ & $30(3.9)$ & $6(0.8)$ \\
\hline $\begin{array}{c}\text { Smear } \\
\text { negative }\end{array}$ & $357(32)$ & $183(51.2)$ & $66(18.5)$ & $37(10.3)$ & $31(8.7)$ & $36(10.1)$ & $4(1.1)$ \\
\hline \multicolumn{8}{|l|}{$\begin{array}{c}\text { HIV } \\
\text { STATUS }\end{array}$} \\
\hline Positive & $256(23)$ & $53(20.7)$ & $106(41.4)$ & $49(19.1)$ & $1(0.4)$ & $46(7.9)$ & $1(0.4)$ \\
\hline $\begin{array}{c}\text { Negative } \\
\text { TB }\end{array}$ & $857(77)$ & $601(70.7)$ & $157(18.3)$ & $15(1.7)$ & $50(5.8)$ & $20(2.3)$ & $7(0.8)$ \\
\hline \multicolumn{8}{|l|}{ CATEGORY } \\
\hline New & $761(68.3)$ & $550(72.2)$ & $127(16.7)$ & $39(5.1)$ & $3(0.4)$ & $38(4.5)$ & $4(0.5)$ \\
\hline Relapse & $70(6.28)$ & $30(42.8)$ & $15(21.4)$ & $7(10)$ & $9(12.8)$ & $8(11.4)$ & $1(1.4)$ \\
\hline Failure & $59(5.3)$ & $25(42.3)$ & $11(18.6)$ & $10(16.9)$ & $8(13.5)$ & $5(8.4)$ & 0 \\
\hline Transfer in & $52(4.6)$ & $22(42.3)$ & $10(19.2)$ & $5(9.6)$ & $7(13.4)$ & $6(11.5)$ & $2(3.8)$ \\
\hline Default & $171(15.3)$ & $32(18.7)$ & $100(58.5)$ & $3(1.7)$ & $24(14)$ & $9(5.2)$ & $3(1.7)$ \\
\hline All & $352(31.6)$ & $109(30.9)$ & 136(38.6) & $25(7.1)$ & $48(13.6)$ & $28(7.9)$ & $6(1.7)$ \\
\hline \multicolumn{8}{|l|}{ Retreatment } \\
\hline \multicolumn{8}{|l|}{ SMOKING } \\
\hline Yes & $596(53.5)$ & $344(57.7)$ & $133(22.3)$ & $39(6.5)$ & $34(5.7)$ & $42(7)$ & $4(0.6)$ \\
\hline No & $517(46.4)$ & $315(60.9)$ & $130(25.1)$ & $25(4.8)$ & $17(3.2)$ & $24(4.6)$ & $6(1.2)$ \\
\hline \multicolumn{8}{|l|}{ ALCOHOL } \\
\hline Yes & $393(35.3)$ & $196(49.8)$ & $115(29.2)$ & $26(6.6)$ & $24(6.1)$ & $30(7.6)$ & $2(0.5)$ \\
\hline No & $720(64.7)$ & $463(64.3)$ & $148(20.5)$ & $38(5.2)$ & $27(3.7)$ & $36(5)$ & $8(1.1)$ \\
\hline
\end{tabular}


Table 5: Factors associated with TB treatment success rate during 2015-17.

\begin{tabular}{|c|c|c|c|c|c|}
\hline Variables & Category & Successfully treated & Unsuccessfully treated & $\begin{array}{c}\text { COR } \\
(95 \% \mathrm{Cl})\end{array}$ & $\begin{array}{c}\text { AOR } \\
(95 \% \mathrm{Cl})\end{array}$ \\
\hline \multirow[t]{2}{*}{ Age (years) } & $\leq 50$ & $490(85.36)$ & $84(14.63)$ & 1.00 & 1.00 \\
\hline & $>50$ & $432(80.14)$ & $107(19.85)$ & $0.69(0.51-0.95)^{* *}$ & $0.47(0.05-0.89)$ \\
\hline \multirow[t]{2}{*}{ Gender } & Male & $588(80.1)$ & $146(19.9)$ & 1.00 & 1.00 \\
\hline & Female & $334(88.1)$ & $45(11.9)$ & $1.84(1.28-2.64)^{\star *}$ & $1.96(1.4-2.52)^{* *}$ \\
\hline \multirow[t]{2}{*}{ Residence } & Urban & $143(86.6)$ & $22(13.3)$ & 1.00 & 1.00 \\
\hline & Rural & $779(82.1)$ & $169(17.8)$ & $0.71(0.43-0.99)$ & $0.66(0.38-0.94)$ \\
\hline \multirow[t]{2}{*}{ Type of TB } & Smear positive & $673(89)$ & $83(10.9)$ & 1.00 & 1.00 \\
\hline & Smear negative & $249(69.7)$ & $108(30.2)$ & $0.28(0.21-0.39)^{* *}$ & $0.35(0.28-0.42)^{* *}$ \\
\hline \multirow[t]{2}{*}{ HIV status } & Positive & $159(62.1)$ & $55(21.5)$ & 1.00 & 1.00 \\
\hline & Negative & $763(89)$ & $92(10.7)$ & $2.87(1.97-4.17)^{* *}$ & $3.01(2.11-3.91)^{* *}$ \\
\hline \multirow[t]{2}{*}{ TB category } & New & $677(88.9)$ & $84(11)$ & 1.00 & 1.00 \\
\hline & All Retreatment & $245(69.6)$ & $107(30.4)$ & $0.28(0.20-0.39)^{* *}$ & $0.34(0.26-0.42)^{* *}$ \\
\hline \multirow[t]{2}{*}{ Smoking } & Yes & $477(80)$ & $119(19.9)$ & 1.00 & 1.00 \\
\hline & No & $445(86)$ & $72(13.9)$ & $1.5(1.12-2.12)^{\star \star}$ & $1.7(1.32-2.08)^{\star *}$ \\
\hline \multirow[t]{2}{*}{ Alcohol } & Yes & $311(79.1)$ & $82(20.8)$ & 1.00 & 1.00 \\
\hline & No & $611(84.8)$ & $109(15.1)$ & $1.47(1.07-2.03)^{\star *}$ & $1.39(0.99-1.79)^{* *}$ \\
\hline
\end{tabular}

** Significant at $P<0.001$; COR: Crude Odds Ratio; AOR: Adjusted Odds Ratio.

Elderly age group patients had higher default (7\%) and failure rates $(7.4 \%)$ in comparison with younger age groups. Male patients were more likely to experience death ( $\mathrm{OR}=1.75,95 \% \mathrm{CI}=0.97-3.16)$ and default $(\mathrm{OR}=2.11,95 \% \mathrm{CI}=1.04-4.27)$ than females. Smear positive patients had lower death rate $(3.9 \%$ vs. $10.1 \% ; P<0.001)$ and failure rate $((2.6 \%$ vs. $8.7 \% ; P<0.001)$ compared to smear negative patients. Patients tested HIV positive had seen significantly unfavorable outcomes in death rate $(\mathrm{OR}=9.17,95 \% \mathrm{CI}=5.31-15.83 ; P<0.001)$ and treatment failure $(\mathrm{OR}=13.3,95 \% \mathrm{CI}=7.31-24.17 ; P<0.0001)$. The default rate $(4.5 \% \mathrm{vs}$. $7.9 \% ; P<0.001)$ is very low in new cases compared to all retreatment cases. Patents with smoking habit and alcohol consumption experienced more death ( $7 \%$ vs. $4.6 \%, 7.6 \%$ vs. $5 \%$; $P<0.001)$ and default $(5.7 \%$ vs. $3.2 \%, 6.1 \%$ vs. $3.7 \% ; P<0.001$ ) as an outcome respectively (Table 4 ).

Multivariable logistic regression analysis showed that female patients had significantly higher treatment success rate than males $(88.1 \%$ vs. 80.1\%; $P<0.001)$. As the age of tuberculosis patients increased, the success rate was decreased. Elderly patients had significantly lower success rate compared to younger age groups. Furthermore, patients from rural area had lower success rate compared to patients from urban areas. However, the difference is not statistically significant $(P=0.179)$ (Table 5).

Smear negative patients had higher risk of unsuccessful treatment outcome than smear negative patients. Poor treatment outcome was found in HIV positive patients. All retreatment cases were less likely to be treated successfully compared to new patients. Smoking habit and alcohol consumption were also considered as risk factors for unfavorable treatment outcome.

\section{DISCUSSION}

According to the WHO report 2017, data showed a global treatment success rate of $83 \%$ for TB. Targeted success rate was at least $85 \%$ among New Sputum Positive (NSP) patients. Treatment success rate among new sputum positive patients and smear negative patients were $89 \%$ and $69.7 \%$ respectively. Our study showed that treatment success rate of all tuberculosis patients was $82.8 \%$ which was close to the targeted

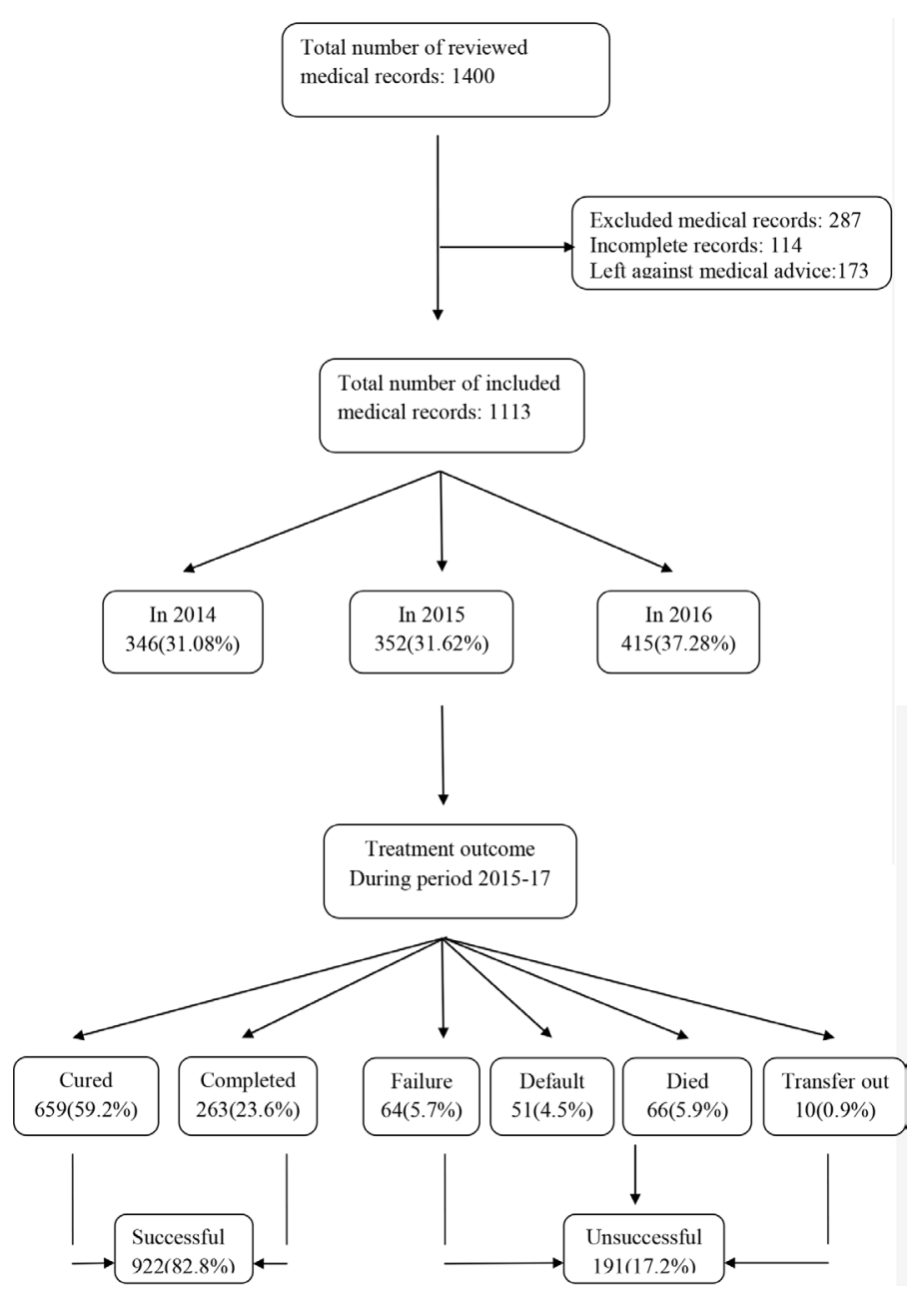

Figure 1: A schematic representation of study design and summary of results. 
success rate. Similar satisfactory results were observed in other studies in India. ${ }^{12,13}$ This might be due to improved adherence of TB patients to treatment. But still there were unsatisfactory treatment success rates. This might be due to failure in identifying the TB treatment failure in early stages. World Health Organization (WHO) recommends diagnosis of TB treatment failure by sputum smear microscopy at 5 months or later during treatment. ${ }^{14}$

Our study showed that treatment success rate increased significantly from 73.9 to $88.9 \%$ during the study period $2015-2017$. The death rate declined from 11.2 to $4.3 \%$ over time. Several other studies demonstrated upward trend in Treatment Success Rate (TSR) and decline in death rate. $^{15}$

Our study also revealed default, death, treatment failure rate and transfer out of $4.5 \%, 5.9 \%, 5.7 \%$ and $0.9 \%$ respectively. These constituted an overall unsuccessful treatment outcome rate of $17.2 \%$ which is lower than other studies (32.2\% and $26 \%$ respectively). ${ }^{16,17}$

In this study, a total of $1113 \mathrm{~TB}$ patients were included. Most of the patients were male similar to other previous studies. ${ }^{18,19}$ This could possibly be due to underutilization of the DOTS service by females or high proportion of males being exposed to the infection in the area.

Unfavorable outcomes such as death, default and treatment failure were more seen in elderly patients. Younger patients were treated successfully than remaining age groups which was observed in previous studies. ${ }^{20}$

Our results were consistent with a previous study in which patients from urban areas $(81.1 \%)$ attending to DOTS had higher success rate compared to patients from rural areas $(66.7 \%) .{ }^{21}$ Poor environmental conditions in the rural areas make patients more vulnerable to tuberculosis and other diseases of poverty and as these populations often are not able to access timely diagnosis or complete the full duration of anti TB treatment, and hence were at risk of unfavorable treatment outcomes including deaths, defaults, failures and drug resistance. Moreover they stop using anti TB medication abruptly once the symptoms were relieved usually after 2 months of initiation of treatment.

Patient was considered smear positive when at least two sputum tests were positive for Acid Fast Bacilli (AFB) by microscopy. If the patient had suggestive symptoms of $\mathrm{TB}$, with at least two specimens which were negative for AFB by microscopy, he was considered smear negative. Success rate was found significantly higher in smear positive patients (89\%) compared to smear negative patients (69.7\%). Our study also revealed that higher death rate and treatment failure occurred with smear negative patients. This is agreed by other studies. ${ }^{22,23}$

The TSR was worse in tuberculosis patients with HIV than without HIV. The study also found that patients with HIV had about 2.5 times higher odds of death and eleven fold higher odds of treatment failure compared to HIV negative TB patients. Studies conducted in Uzbekistan and Ethiopia showed the unfavorable treatment outcomes in TB with HIV patients. ${ }^{24,25}$

The TSR among re-treatment cases was much lower than new smear positive patients. This was supported by previous studies where re-treatment cases were significantly associated with unsuccessful treatment outcome. WHO Guidelines for the programmatic management of drug-resistant tuberculosis. ${ }^{26,27}$ Moreover, the current study revealed that there was a higher default and death rate among re-treatment compared to the new cases.

Both smoking and alcoholism were considered as important risk factors for unsuccessful treatment outcomes like treatment failure, death rate and default rate. Our result is supported by earlier studies..$^{28,29}$

\section{CONCLUSION}

It is possible to achieve the recommended WHO target which is $85 \%$ for newly diagnosed smear positive patients. There is significant increase of TSR over the study period. Implementing the DOTS strategy proved the satisfactory success rate in the hospital across three years. The unsuccessful treatment outcome was significantly associated with gender, HIV status, re-treatment, smear negative, smoking and alcoholism. Regular follow up and creating awareness among patients with poor socioeconomic background is important. In addition, more care should be taken for smear negative and HIV positive patients by regular sputum follow up tests.

\section{Limitations}

Although study focused on various socio-cultural factors which affect the outcome of treatment, details of income of patients could not be retrieved from the register. We could not assess the degree of drug resistance due to unavailability of drug susceptibility tests. Some patients were transferred out each year due to unavailability of DOTS near to their residence.

\section{ACKNOWLEDGEMENT}

Authors are thankful to the head of Govt. ID Hospital, Guntur, India and staff members of TB clinic for providing support and facilitating the data collection.

\section{CONFLICT OF INTEREST}

The authors declare no conflict of interest.

\section{ABBREVIATIONS}

AOR: Adjusted odds ratio; COR: Crude odds ratio; DOTS: Directly Observed Treatment Short-Course; Govt. ID: Government Infectious Disease; RNTCP: Revised National Tuberculosis Control Program; TSR: Treatment Success Rate; WHO: World Health Organization.

\section{REFERENCES}

1. World Health Organization (WHO): Global tuberculosis report 2016. WHO report. WHO/HTM/TB/2016.13.

2. World Health Organization (WHO): Global tuberculosis report 2017. WHO report. WHO/HTM/TB/2017.23.

3. Williams BG, Granich R, Chauhan LS, Dharmshaktu NS, Dye C. The impact of HIV/AIDS on the control of tuberculosis in India. Proc Natl Acad Sci USA. 2005;102(27):9619-24

4. Tesfahuneygn G, Medhin G, Legesse M. Adherence to Anti-tuberculosis treatment and treatment outcomes among tuberculosis patients in Alamata District, Northeast Ethiopia. BMC Res Notes. 2015;8(1):1-11.

5. Central TB Division. TB India 2004, RNTCP Status Report (2004) Directorate General Health Services, Ministry of Health and Family Welfare, New Delhi, Government of India. http://www.tbcindia.org/pdfs/tbindia2004.pdf.

6. Shargie EB, Lindtjorn B. DOTS improves treatment outcomes and service coverage for tuberculosis in South Ethiopia: a retrospective trend analysis. BMC Public Health. 2005;5(1):1-11.

7. Revised National Tuberculosis Control Programme. National Health Portal Of India. www.nhp.gov.in. Retrieved 2017-07-18.

8. RNTCP Government of India TB Treatment Education and Care. TB Facts.org. Retrieved 2017-07-18.

9. National AIDS Control Organization and Central TB Division. National framework for joint TB/HIV collaborative activities. New Delhi: National AIDS Control Organization and Central TB Division, Ministry of Health and Family Welfare, Government of India; October. 2009.

10. National AIDS Control Organization and National Institute of Medical Statistic I. Technical report, India HIV estimates-2006: ICMR. 2006

11. WHO. Treatment of tuberculosis: guidelines for national treatment programmes; 2004.

12. Jackson C, Stagg HR, Doshi A, Pan D, Sinha A, Batra R, et al. Tuberculosis treatment outcomes among disadvantaged patients in India. Public Health Action. 2017;7(2):134-40.

13. Ramachandran G, Kumar AH, Chandrasekaran V, Kannan T, Vijayalakshmi R, 
Dusthackeer VA, et al. Factors influencing tuberculosis treatment outcome in adult patients treated with thrice-weekly regimens in India. Antimicrob Agents Chemother. 2017:61(5):1-12.

14. Harries A, Maher D, Graham S. Management of patients with tuberculosis. In: TB/HIV a clinical manual, Geneva: World Health Organisation. $2^{\text {nd }}$ ed. 2004;111-5.

15. Hamusse SD, Demissie M, Teshome D, Lindtjørn B. Fifteen-year trend in treatment outcomes among patients with pulmonary smear-positive tuberculosis and its determinants in Arsi Zone, Central Ethiopia. Glob Health Action. 2014; 7(1):25382.

16. Atif M, Anwar Z, Fatima RK, Malik I, Asghar S, Scahill S. Analysis of tuberculosis treatment outcomes among pulmonary tuberculosis patients in Bahawalpur, Pakistan. BMC Res Notes. 2018;11(1):1-6.

17. Wondale B, Medihn G, Teklu T, Mersha W, Tamirat M, Ameni G. A retrospective study on tuberculosis treatment outcomes at Jinka General Hospital, southern Ethiopia. BMC Res Notes. 2017;10(1):1-7.

18. Datiko DG, Lindtjorn B. Tuberculosis recurrence in smear-positive patients cured under DOTS in southern Ethiopia: retrospective cohort study. BMC Public Health. 2009;9(1):1-5.

19. Venkatapraveen A, Rampure M, Patil N, Hinchageri SS, Lakshmi DP. Assessment of clinical pharmacist intervention to improve compliance and health care outcomes of tuberculosis patients. Der Pharmacia Lettre. 2012;4(3):931-7.

20. Patra S, Lukhmana S, Tayler K, Kannan AT, Satyanarayana S, Enarson DA, et al. Profile and treatment outcomes of elderly patients with tuberculosis in Delhi, India: implications for their management. Trans R Soc Trop Med Hyg. 2013;107(12):763-8.

21. Musaazi J, Kiragga AN, Castelnuovo B, Kambugu A, Bradley J, Rehman AM. Tuberculosis treatment success among rural and urban Ugandans living with
HIV: a retrospective study. Public Health Action. 2017;7(2):100-9.

22. Vasudevan K, Jayakumar N, Gnanasekaran D. Smear conversion, treatment outcomes and the time of default in registered tuberculosis patients on RNTCP DOTS in Puducherry, Southern India. J Clin Diagn Res. 2014; 8(10):JC05-8.

23. Gebrezgabiher G, Romha G, Ejeta E, Asebe G, Zemene E, Ameni G, et al. Treatment outcome of tuberculosis patients under directly observed treatment short course and factors affecting outcome in Southern Ethiopia: a five-year retrospective study. PloS One. 2016;11(2):e0150560.

24. Gadoev J, Asadov D, Tillashaykhov M, Tayler-Smith K, Isaakidis P, Dadu A, et al. Factors associated with unfavorable treatment outcomes in new and previously treated TB patients in Uzbekistan: a five year countrywide study. PloS One. 2015;10(6):e0128907.

25. Gebremariam G, Asmamaw G, Hussen M, Hailemariam MZ, Asegu D, Astatkie A, et al. Impact of HIV status on treatment outcome of tuberculosis patients registered at Arsi Negele Health Center, Southern Ethiopia: a six year retrospective study. PloS One. 2016;11(4):e0153239.

26. World Health Organization. Guidelines for the programmatic management of drug-resistant tuberculosis. Geneva WHO. 2008.

27. Shah NS, Wright A, Bai GH, Barrera L, Boulahbal F, Martín-Casabona N, et al. Worldwide emergence of extensively drug-resistant tuberculosis. Emerg Infect Dis. 2007;13(3):380-7.

28. Leung CC, Yew WW, Chan CK, Chang KC, Law WS, Lee SN, et al. Smoking adversely affects treatment response, outcome and relapse in tuberculosis. Eur Respir J. 2005;45(3):738-45

29. Myers B, Bouton TC, Ragan EJ, White LF, Mcllleron H, Theron D, et al. Impact of alcohol consumption on tuberculosis treatment outcomes: prospective longitudinal cohort study protocol. BMC Infect Dis. 2018;18(1):1-9.

Article History: Submission Date : 28-08-2018; Revised Date : 04-10-2018; Acceptance Date : 30-11-2018.

Cite this article: Prudhivi R, Challa SR, Rao MVB, Veena G, Roa BN, Narne HM. Assessment of Success Rate of Directly Observed Treatment Short-Course (DOTS) in Tuberculosis Patients of South India. J Young Pharm. 2019;11(1):67-72. 